\title{
Hepatitis C
}

\section{Folgekomplikationen verhindert, Morbidität gesenkt}

_ Allein in Deutschland könnten Modellrechnungen zufolge bis zum Jahr 2030 lediglich durch die Steigerung der therapeutischen Effektivität der Anteil der virämischen, $\mathrm{HCV}$-infizierten Patienten sowie leberbedingter Todesfälle um jeweils $40 \%$ und kompensierte bzw. dekompensierte Zirrhosen um 55\% gesenkt werden [Wedemeyer H et al. J Viral Hepatol. 2014:21:60-89 ]. Durch eine Verdopplung der aktuellen Therapierate würden in absehbarer Zeit sechs von sieben HCV-assoziierten Zirrhosen vermieden, betonte Prof. Stefan Zeuzem, Frankfurt/Main.

Als bislang erster und einziger frei kombinierbarer NS5A-Replikationskomplexinhibitor ist Daclatasvir (Daklin$\mathrm{za}^{\varpi}$ ) zur Behandlung der chronischen HCV-Infektion beim Erwachsenen ver- fügbar: Der NS5A-Inhibitor ist pangenotypisch wirksam, sodass er in Kombination mit anderen antiviralen Arzneimitteln prinzipiell gegen alle $\mathrm{HCV}$ Genotypen (HCV-GT) 1-6 eingesetzt werden kann. Patienten mit HCV-GT 1-3 hatten in der zulassungsrelevanten Phase-II-Studie (AI444-040) mit Daclatasvir/Sofosbuvir Heilungsraten von bis $\mathrm{zu} 100 \%$ erreicht.

\section{Phase-III-Daten mit Daclatasvir: ALLY- Daten vervollständigt}

Im Studienprogramm ALLY wurde Daclatasvir plus Sofosbuvir inzwischen auch in Phase-III-Studien evaluiert [Nelson DR et al. Hepatology. 2015;61: 1127-35; Poordad F et al. EASL 2015 (Oral Presentation L08); Wyles D et al. CROI 2015,
Abstract 151LB]: Untersucht wurden $\mathrm{Pa}$ tienten aller HCV-Genotypen mit und ohne Vortherapie, einschließlich Patienten mit Leberzirrhose sowie nach Transplantation (ALLY-1), mit HIV-/HCV-Koinfektion (ALLY-2) oder HCV-GT3-Infektion (ALLY-3). Dabei hätten Patienten mit HCV-GT3-Infektion bislang eine besonders schwer behandelbare Patientengruppe repräsentiert, so Zeuzem: GT3 sei u.a. mit einem höheren Risiko für eine Steatosis, progrediente Fibrose, ein hepatozelluläres Karzinom (HCC) und mit einer höherenMortalität assoziiert als andere HCV-Genotypen.

Dr. Yuri Sankawa

- Post-EASL-Pressegespräch "Gegenwart und Zukunft der HCV-Therapie: Ein EASL-Update zu Daclatasvir (Daklinza $\left.{ }^{\circ}\right)^{\prime \prime}$ Frankfurt/M., Mai 2015 (Veranstalter: Bristol-Myers Squibb)

\section{Kompressionstherapie}

\section{Bandagensystem erleichtert Selbstmanagement}

\begin{abstract}
Kompressionsmaßnahmen sind einer der tragenden Pfeiler in der Therapie von venösen Beinulzera. Wie Prof. Joachim Dissemond, Essen, erläuterte, greift die Kompression über die Einengung der dilatierten Venen und über die Steigerung
\end{abstract}

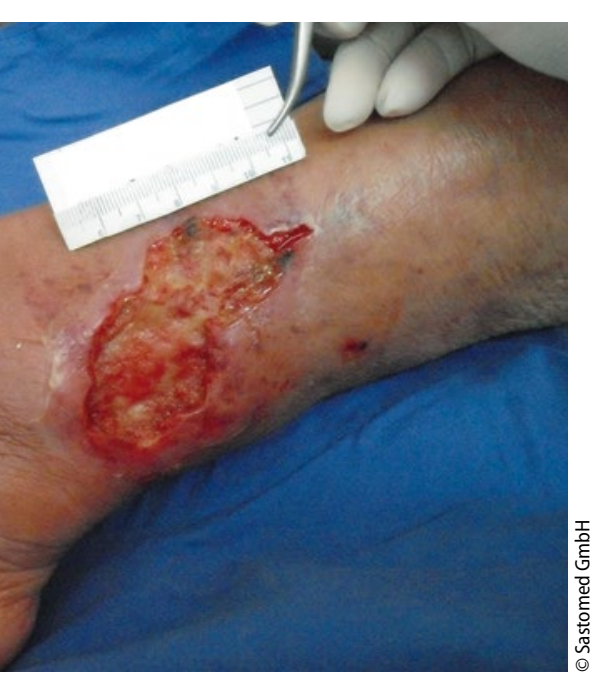

Venöses Ulclus venosum - hier ist eine konsequente Therapie gefragt! des Gewebedrucks kausal in das Krankheitsgeschehen ein.

Das Anlegen eines Kompressionsverbands bedarf einer gewissen Übung und Erfahrung, damit der Verband weder zu fest noch zu locker sitzt und genau den erforderlichen Druck auf das Bein ausübt. Dass dies auch erfahrenen Pflegekräften oft nicht gelingt, hat eine Studie gezeigt, in der unter Kompressionsverbänden, die ambulant zur Behandlung des Ulcus cruris angelegt worden waren, Druckwerte zwischen 11 und $80 \mathrm{mmHg}$ gemessen wurden [Zarchi K, Jemec GB, 2014]. Die gewünschten optimalen Druckwerte zwischen 30 und $50 \mathrm{mmHg}$ wurden im günstigsten Fall bei Verwendung von Zweikomponenten-Systemen von nur $27 \%$ der Pflegekräfte erreicht.

Verschiedene Untersuchungen haben ergänzend belegt, dass lediglich ein geringer Teil der Patienten mit entsprechendem Bedarf auch tatsächlich eine Kompressionstherapie verordnet bekommt [Rabe E et al. 2013]. „Aus diesen
Beobachtungen lässt sich schließen, dass das Wissen um diese physikalische Behandlungsmethode und das diesbezügliche Können sowohl bei Pflegekräften als auch bei Ärzten zunehmend verloren geht“, schlussfolgerte Dissemond.

\section{Einfache Kompressionsversorgung}

Mit circaid ${ }^{\circledast}$ juxtacures ${ }^{\circledast}$ ist in Deutschland jetzt ein KompressionsbandagenSystem zur Behandlung von venösen Ulzera verfügbar, zu dessen wichtigsten Pluspunkten seine einfache Handhabung zählt. Das Anlegen der Versorgung ist so unkompliziert, dass diese Aufgabe leicht vom Patienten selbst übernommen werden kann. Das circaid ${ }^{\circledast}$ Built-In-Pressure ${ }^{\oplus}$-System (BPS) ermöglicht das eigenständige Einstellen des verordneten Kompressionsniveaus sowie dessen Nachjustierung an sich verändernde Beinumfänge.

\section{Dr. Silke Wedekind}

- Expertenworkshop „Kompression“; Bayreuth, Juni 2015 (Veranstalter: Medical Data Institute) 